\title{
Virtual Reality Educational Tool for Connecting Chemistry Lecture with Remote Laboratory Demonstrations
}

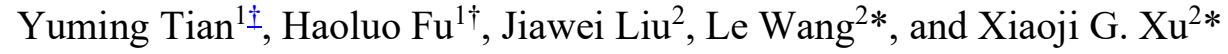 \\ ${ }^{1}$ Department of Computer Science and Engineering, Lehigh University, 113 Research Drive, \\ Bethlehem, PA, 18015 \\ ${ }^{2}$ Department of Chemistry, Lehigh University, 6 E. Packer Ave., Bethlehem, PA, 18015
}

Email: lew314@lehigh.edu andxgx214@lehigh.edu

Note ${ }^{\dagger}$ : These authors contributed equally.

\begin{abstract}
Providing access to up-to-date and expensive instruments for a large classroom is challenging for courses of analytical chemistry and physical chemistry, where knowledge on analytical methods or demonstration of phenomena is associated with a specialized instrument. In this technology report, we present our solution to this challenge: a virtual reality (VR) chemical educational tool that provides in situ user experience on research equipment to a large classroom. The VR educational tool consists of a VR camera in the laboratory attached to a teaching assistant who performs the experiment and many virtual reality playing devices in a classroom watched by students. The experience of experimenting is shared with the students in the classroom. Moreover, the VR educational tool can also provide an immersive experience on laboratory demonstrations to the general audience for outreach, without the constraint from safety concerns. Further utilization of the VR educational tool is expected to remove the barrier between the lecture room and the laboratory and enable new pedagogical methods for lectures in STEM education.
\end{abstract}

Keywords: Upper-division undergraduate, laboratory instruction, analytical chemistry, physical chemistry, distance learning/self instruction, computer-based learning, Internet/web-based learning, laboratory equipment/apparatus

\section{Introduction}

Modern analytical and physical chemistry research evolve with the emergence of new research tools and scientific instruments. The development of various kinds of microscopes, spectrometers, and chromatography have revolutionized how chemical imaging, analysis, and separations are performed in the past decades..$^{1-3}$ The chemical education on analytical and physical chemistry should keep pace with the innovations of modern scientific instruments. However, those instruments are often expensive to purchase, maintain, and delicate to operate. Very seldom can an academic institute afford a fleet of expensive and up-to-date research instruments just for educational purposes. Consequently, the design on the curriculum of analytical chemistry is limited by the access and availability of the scientific instruments, rather than keeping pace with the latest development. How to overcome this dilemma between limited access to scientific instruments and the need for education on advanced content that requires scientific instruments? In this technology report, we tackle this challenge with the development of virtual reality (VR) 
chemical education tool that connects scientific instruments with a large classroom through VR links. Virtual reality technology provides an immersive experience to users of locations that they are unable to be physically present. ${ }^{4}$ In the past, VR technology has been utilized to deliver a virtual field trip to an oversea site for environmental chemistry lectures. ${ }^{5}$ VR technology was also applied in organic chemistry lectures to let students experience molecular structures and motions. ${ }^{6}$ In our development, we further implement VR technology in real-time and include interactions between students, instructors, and teaching assistants for teaching chemistry courses.

\section{The design of the virtual reality chemical educational tool}

The implementation of the VR educational tool is to connect two operational sites via the Internet: the proxy site is the laboratory for instrument or experiment demonstration; the lecture site is the classroom with instructor and students. The students are in the classroom wearing VR goggles. A teaching assistant (TA) is on the laboratory site, operating the scientific instrument, and wearing a VR camera with an image-stabilization mechanism. The VR camera captures 360 degrees the operations performed by the TA and passes the VR video to a compact laptop computer. The computer is connected to a server ("WebRTC" - Web Real-Time Communication) in the cloud. The server streams the VR videos from the laptop computer with the VR camera to the VR goggles (e.g. Occulus Go) in the classroom site. The same VR video is also accessible on mobile phones for smart-phone-based VR goggles, as well as on personal computers with an Internet browser (e.g., Chrome). The students will observe all the operations on the proxy site of the laboratory by the TA. The TA also wears a Bluetooth earphone with a microphone (e.g. Apple Airpod with iPhone). The TA in the laboratory is connected with the instructor via voice line, e.g. wireless telephone, so that the TA can perform operations at the request of the instructor. Note that the earphone headset is preferred over speaker mode to reduce the amplification of echoes. The instructor in the classroom gives lectures and obtains feedback from the students. If students are curious about a particular operation, the instructor will discuss with the students and ask TA to operate on the proxy site. Figure 1 illustrates the essential elements of the VR educational tool. The detailed construction of the VR educational tool is described in the following sections.

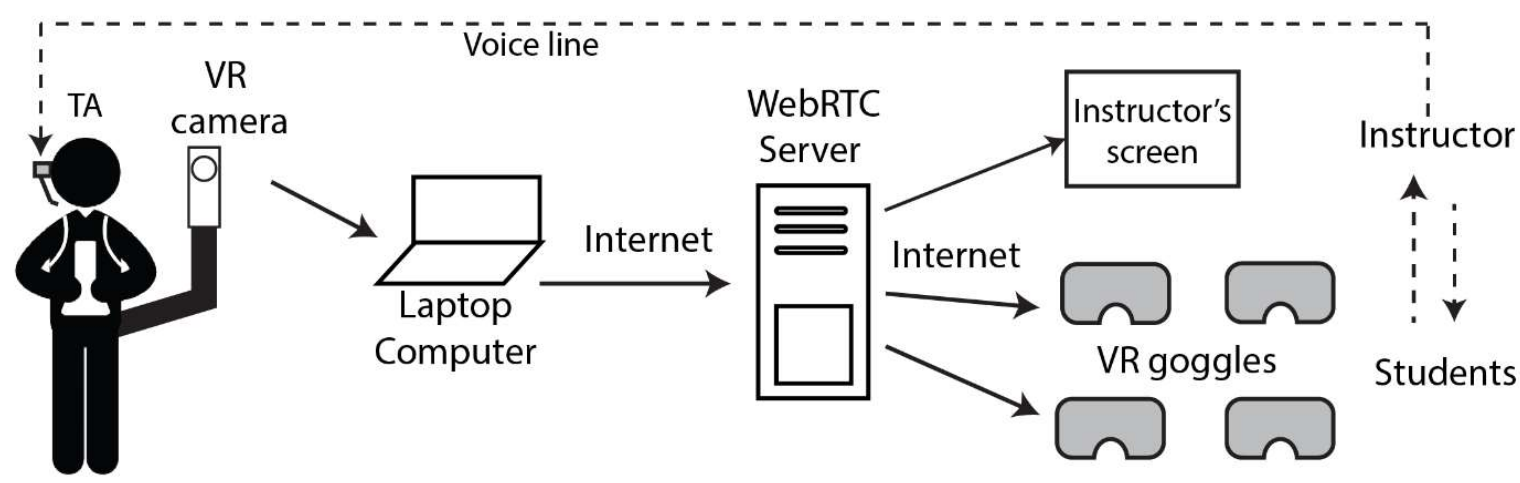

Figure 1. The architecture of the VR chemical educational tool. It consists of a VR camera controlled by the TA and a laptop that streams VR videos to a WebRTC server. The WebRTC server broadcasts VR streams to VR goggles that are worn by students, and the streams are also displayed on a screen accessible to the instructor. A voice line between the instructor and the TA is used to pass the instructions.

\section{VR camera and stabilization}


The VR camera (Ricoh Theta) is attached to a camera vest worn by the TA. The purpose of the design is to create an always-accompanying camera without holding the camera by hand so that the TA can perform experiments. We use a gimbal camera stabilization platform (G6 Plus FeiYu Tech) to avoid small vibrations or unwanted tilt or pitch caused by the body motion of the TA. Without the gimbal stabilization, vibrations or rotation of the video capture may cause discomfort to the watcher on the classroom site. The attachment arm of the camera vest can also adjust the height and relative positions, so the TA can find a good streaming position to reveal details of the experiment.

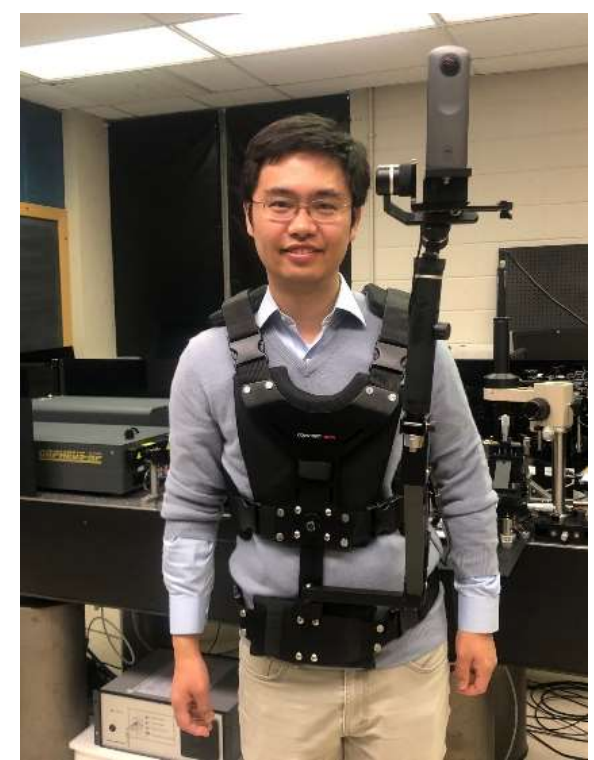

Figure 2. Mounted VR camera on a vest with gimbal vibrational stabilization.

\section{VR streaming platform - WebRTC}

The essential component to implement the VR educational tool is an easy-to-use VR steaming platform that allows educators to broadcast lectures and experiments in real-time. In our development, we built a web application that requires no installation and can run on most devices with an Internet browser. The detailed setup guide can be found in Supporting Information Note S1. This tutorial shows you how to broadcast or watch live stream using the WebRTC platform we built. The VR streaming platform should also have low latency so that interactions between the TA site and the classroom site are without time delay. Currently, available VR streaming platforms, such as YouTube, have latency about $5-10$ seconds. Such a long latency makes the interaction between the TA site and the classroom site difficult. On the other hand, most real-time communication tools, such as Skype, only stream videos in low resolution and does not support VR. In this case, we created our streaming platform based on WebRTC technology. The server we built can stream videos with latency lower than $500 \mathrm{~ms}$. Also, we used JavaScript plugin (video-js-panorama) to display VR videos nicely on 2D screens. Even if users do not have a VR headset, they can watch the broadcast using their laptops or smartphones in a browser. The source code of the WebRTC is available at https://github.com/maizerrr/webrtc_streaming and is 
included in Supporting Information Note S2. The broadcasters can build their server by following the procedures in Note S2. In the WebRTC platform, we also created the capability of voice line link via WebRTC server between the TA and the instructor. Alternatively, a regular voice line through a mobile phone or Skype between the TA and the instructor is sufficient to establish the connection. A separate voice link will reduce the load of the WebRTC server. In the classroom, students can use VR goggles (Occulus Go) to receive the VR stream. The VR stream can also be accessed with a regular web browser (e.g., Google Chrome) if VR goggles are not available. A web browser is used by the instructor in the classroom to access the video stream at the same time.

\section{VR streaming platform - commercial streaming platforms}

An alternative to WebRTC is to use commercially available streaming platforms, which are convenient and accessible for educators without programming expertise. We have tested that the streaming platform Twitch.com is suitable for VR educational tools. Twitch allows VR streaming from VR camera to goggles. The streaming platform via Twitch can be set up by OBS (Open Broadcaster Software) studio with the streaming function at the VR capture site by the TA (instructions included in Supporting Information Note S3). Watching the VR stream is achieved through the Twitch app on smartphones or VR goggles. The commercial stream platforms provide a stable backup to the WebRTC approach. For instance, if the WiFi network connection of the school fails, instructors can still use data connection from a wireless network to broadcast. However, the disadvantage of Twitch is the relatively high latency: up to five seconds delay from the VR capture side to the VR goggles. The instructor of the lecture has to adapt to the five-second delay of VR streaming and adjusts the pace appropriately. In a regular laboratory demonstration, a five-second delay is acceptable. The delay is expected to be reduced in the future as communication technology improves.

\section{Results}

The first experiment we demonstrated with the VR educational tool is the Briggs-Rauscher reaction, ${ }^{7-8}$ which is one of the well-known chemical oscillating reactions. The initial aqueous solution contains hydrogen peroxide $\left(\mathrm{H}_{2} \mathrm{O}_{2}\right)$, potassium iodate $\left(\mathrm{KIO}_{3}^{-}\right)$, sulfuric acid $\left(\mathrm{H}_{2} \mathrm{SO}_{4}\right)$, malonic acid $\left(\mathrm{CH}_{2}(\mathrm{COOH})_{2}\right)$, manganese sulfate monohydrate $\left(\mathrm{MnSO}_{4} \cdot \mathrm{H}_{2} \mathrm{O}\right)$, and starch as the indicator. The color will change from colorless to amber and then dark blue, due to the significant fluctuation of the concentration of iodine. The chemical oscillation of the color swing of the Briggs-Rauscher reaction makes it an ideal example of a demonstration to the general public. However, since the reaction involves the production of elemental iodine that can cause respiratory issues when inhaled, the reaction should be held in a fume hood in a laboratory, rather than in the lecture room. An onsite demonstration may be restricted due to this chemical safety concern. On the other hand, the utilization of a VR educational tool avoids the safety concerns and delivers the demonstration for classroom settings. 
(a)

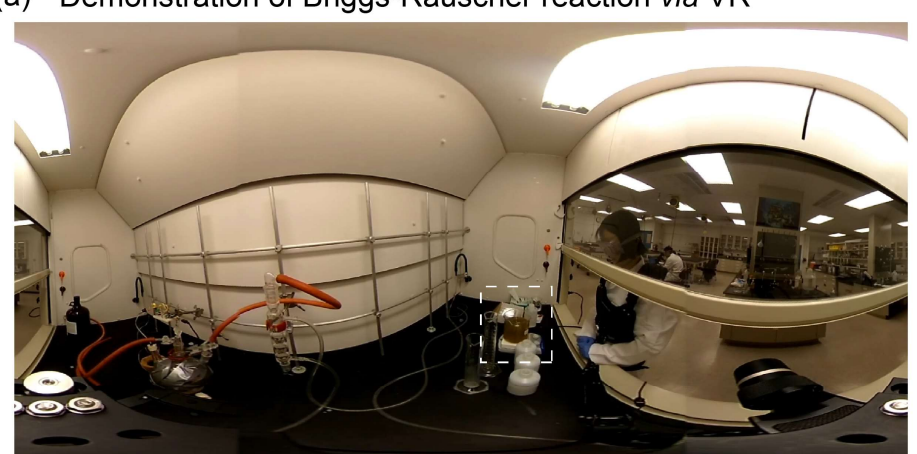

(b)

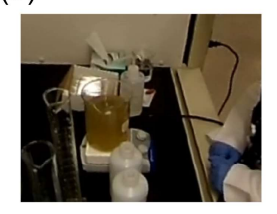

(c)

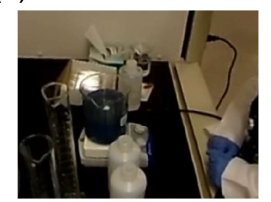

(e) Zoomed in of details

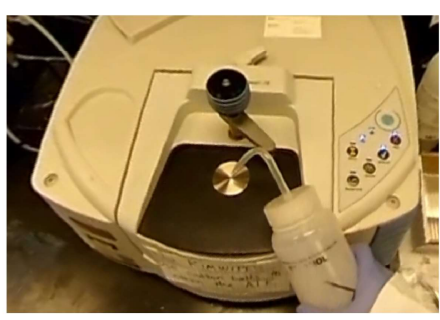

Figure 3. (a) VR capture of the demonstration of Briggs-Rauscher reaction. (b-c) Characteristic color oscillations of Briggs-Rauscher reaction from a zoomed-in video between amber and dark blue color. (d) VR capture of the demonstration of a FTIR spectrometer. (e) The key details of the instrument operation are zoomed in for illustration.

The second demonstration is to show how a Fourier-transform infrared (FTIR) spectrometer works. FTIR spectroscopy has been widely used for chemical identification of materials. ${ }^{9-10}$ Learning FTIR spectroscopy is important for analytical chemistry education. We demonstrated the collection of a FTIR spectrum on acetone (99.5\%, BDH Chemicals) with a FTIR spectrometer (Thermo Nicolet iS10 FT-IR spectrometer) and performed basic spectrum analysis with the software. Students can learn how to operate a FTIR spectrometer and extract information from an IR spectrum without waiting in line to do the actual experiment. Similar demonstrations can be carried out for other instruments for educational purposes, such as the demonstration of an atomic force microscope to students.

\section{Discussion}

The usage of the VR educational tool is not limited to chemical education for analytical and physical chemistry. Related STEM disciplines that need the demonstration of a limited quantity of advanced research instruments to a large group of students can also adopt the VR educational tool. For example, the subject of nonlinear optics in physics requires high peak power (picosecond or femtosecond) laser systems that are expensive to purchase and maintain. To accommodate a large group of students in a tight space of an optical laboratory may also raise laser safety concerns. Those demonstrations of nonlinear and ultrafast spectroscopy can be solved with the VR education tool. 
VR-assisted teaching may enable a new type of pedagogical approach for physical and analytical chemistry that integrates lecture and lab sessions. Immediately after introducing a new physical phenomenon or an analytical technique in the classroom, the remote VR demonstrations will follow. For example, the collapse of wave functions, an abstract concept in quantum mechanics, can be demonstrated with the polarization state of Helium-Neon laser with polarizers and wave plates by the TA in the laboratory right after the concept is introduced in the classroom. This timely VR experience will solidify the learning outcome. For analytical chemistry, the operation of an advanced instrument, such as a liquid chromatography-mass spectrometry (LC-MS), can be demonstrated to the students in the classroom after the introduction of the corresponding analytical techniques. The VR educational tool will scale well with the number of students. In essence, additional students simply require more VR goggles rather than more instruments in the laboratory or additional laboratory preparation by TAs.

Another application of the VR educational tool is to provide teaching service during the special time that social distancing is required. For example, the COVID-19 outbreak in 2020 momentarily shifts the lectures online to avoid gathering a large group of students in a classroom or a laboratory. The online teaching of laboratory courses that involves interaction between students and instruments is difficult without access to the instrument. The VR educational tool can be used to connect students at home to experiment demonstrations in the lab by the TA. The classroom site for the VR educational tool is not necessarily limited to a physical classroom; instead, the interaction between students and the instructor can be provided by an online classroom to satisfy the requirement of social distancing.

The VR educational tool can be integrated into VR lab demonstrations, ${ }^{11}$ such as using 3D modeling to create VR settings in computers for demonstration of experimental procedures or instruments before the actual laboratory session starts. In this case, students will be better prepared for performing lab sessions. It also allows teaching advanced STEM courses for remote areas where access to the actual experimental equipment is limited. The VR educational tool will be an equalizing technology for the disparity of educational resources due to geographic restrictions or the level of social development. The VR educational tool can also be used for outreach to K12 schools by laboratories of higher education institutes.

\section{Notes}

The authors declare no competing financial interests.

\section{Acknowledgment}

X. G. X and L. W. would like to thank the support from the National Science Foundation, award number CHE 1847765. Y. T. and H. F. would like to thank the summer research support from the Mountaintop project of Lehigh University.

\section{References}

1. Council, N. R. Visualizing Chemistry: The Progress and Promise of Advanced Chemical Imaging; National Academies Press, 2006. 
2. Willard, H. H.; Merritt Jr, L. L.; Dean, J. A.; Settle Jr, F. A. Instrumental Methods of Analysis. 1988.

3. Rouessac, F.; Rouessac, A. Chemical Analysis: Modern Instrumentation Methods and Techniques; John Wiley \& Sons, 2013.

4. Sherman, W. R.; Craig, A. B. Understanding Virtual Reality: Interface, Application, and Design; Elsevier, 2002.

5. Fung, F. M.; Choo, W. Y.; Ardisara, A.; Zimmermann, C. D.; Watts, S.; Koscielniak, T.; Blanc, E.; Coumoul, X.; Dumke, R. Applying a Virtual Reality Platform in Environmental Chemistry Education to Conduct a Field Trip to an Overseas Site. J. Chem. Educ. 2019, 96(2), 382-386.

6. Ferrell, J. B.; Campbell, J. P.; McCarthy, D. R.; McKay, K. T.; Hensinger, M.; Srinivasan, R.; Zhao, X.; Wurthmann, A.; Li, J.; Schneebeli, S. T. Chemical Exploration with Virtual Reality in Organic Teaching Laboratories. J. Chem. Educ. 2019, 96(9), 1961-1966.

7. Briggs, T. S.; Rauscher, W. C. An Oscillating Iodine Clock. J. Chem. Educ. 1973, 50(7), 496.

8. Furrow, S. D. A Modified Recipe and Variations for the Briggs-Rauscher Oscillating Reaction. J. Chem. Educ. 2012, 89(11), 1421-1424.

9. Perkins, W. Fourier Transform-Infrared Spectroscopy: Part I. Instrumentation. J. Chem. Educ. 1986, 63(1), A5.

10. Vasanthan, N. Crystallinity Determination of Nylon 66 by Density Measurement and Fourier Transform Infrared (FTIR) Spectroscopy. J. Chem. Educ. 2012, 89(3), 387-390.

11. Bibic, L.; Druskis, J.; Walpole, S.; Angulo, J.; Stokes, L. Bug Off Pain: An Educational Virtual Reality Game on Spider Venoms and Chronic Pain for Public Engagement. J. Chem. Educ. 2019, 96(7), 1486-1490.

\title{
Supporting Information
}

\section{Virtual Reality Educational Tool for Connecting Chemistry Lecture with Remote}

\section{Laboratory Demonstrations}

\author{
Yuming Tian ${ }^{1 \dagger}$, Haoluo $\mathrm{Fu}^{1 \dagger}$, Jiawei $\mathrm{Liu}^{2}$, Le Wang ${ }^{2 *}$, and Xiaoji G. Xu${ }^{2 *}$ \\ ${ }^{1}$ Department of Computer Science and Engineering, Lehigh University, 113 Research Drive, \\ Bethlehem, PA, 18015 \\ ${ }^{2}$ Department of Chemistry, Lehigh University, 6 E. Packer Ave., Bethlehem, PA, 18015
}

\section{Note S1. Instructions for using WebRTC for broadcasters and viewers}

\section{Step 1: Join the chat room}

Visit https://webrtc.maizer.pw, which is a real-time communication platform we built, with a supported browser (Google Chrome, Firefox, Safari). The website will ask you to enter the name of the chat room you want to join. If the server cannot find a room match the name provided, it 
will create a new chat room. To start broadcasting, the broadcaster should always create a new chat room.

\section{Step 2: Broadcaster \& viewers}

The teaching assistant (TA) in the laboratory site will first create a chat room and become the broadcaster. The https://webrtc.maizer.pw website will ask for permission to access both the VR camera of the TA (e.g., in our case, the Ricoh Theta V) and the microphone of the VR camera. The students and instructor in the classroom site will join the chat room created by the TA and become viewers. The website will only ask for permission to access the microphones of the students and the instructors. The purpose of the access to students' and instructor's microphone is to serve as a backup, should the voice line between the instructor and the TA become unusable. In operation, the students should mute their microphones while in VR streaming.

Students in the classroom site will configure the built-in browser of the goggle into VR mode. The instructor should use a regular browser to access the VR stream, preferably projected on a screen in the classroom.

\section{Step 3: Video stream}

The broadcaster (the TA in the laboratory site) will share the video stream captured by the web camera with every viewer (students and the instructor in the classroom site) in the chat room. On the web page, both the broadcaster and viewers will see the same VR video (which is captured by the VR camera of the TA). If video is not played automatically, click the play button of the web browser of the VR goggle.

\section{Step 4: Audio stream}

Live audio chat is automatically enabled from the TA to the students and the instructor. The software architecture allows each viewer (students and the instructor) to chat with the broadcaster (TA). In typical operation, only the chat between the instructor and the TA is active.

\section{Step 5: Leave the chat room}

After the utilization of the VR educational tool, users (TA, students, and the instructor) can leave the chat room simply by closing the browser tab. If there is no active user in the chat room, it will automatically be released and deleted. It is recommended that every user should leave the chat room after the usage of the VR educational tool so that the server can free chat rooms that are no longer active.

\section{Note S2. Procedures for building a WebRTC server}

The project https://github.com/maizerrr/WebRTC streaming was developed based on Google WebRTC code labs[1] and the 360 video player was created with Videojs-panorama[2]. The following are the instructions on how to build a WebRTC server.

\section{Required dependencies}


$\underline{\text { nginx }}, \underline{\text { node.js }}, \underline{\text { screen }}$

\section{Step 1: Create a directory for the website}

To create the root directory for the web, you can run "mkdir/home/wwwroot/" in the terminal of your server. Then use "cd /home/wwwroot/" to go to the directory that has just been created.

\section{Step 2: Download repository from Github}

Under the root directory of your website, run "git clone

https://github.com/maizerrr/WebRTC_streaming.git". If git command is not found, run "yum -y install git" and try again. If the repository is successfully cloned, you can see all the source files in the current directory by entering "ls".

\section{Step 3: Start the service}

Before you can run the code, you need to run "npm install" to install or update dependencies. Then, use "screen -S WebRTC" to create a new screen called 'WebRTC'. This allows your WebRTC service to run in the background. Otherwise, WebRTC would terminate immediately after you close the ssh connection. In the screen created, run "node index.js". To detach from the current screen, press "ctrl" and "A" at the same time, then press "D".

\section{Step 4: Configure https encryption}

After detached from the screen, your WebRTC service will be running in the backend. You can check this by entering "http://:8443" in your browser. Be aware that WebRTC is not ready yet since it requires https encryption. So as long as you do not get a "404 not found" page, your server is working.

To enable https, you need to use nginx. First, edit /etc/nginx/nginx.conf. Find a section called 'server' which listens 'port 80' (there are two server sections). Put your domain name in the 'server_name' attribute. Save nginx.conf and enter "nginx -s reload" to update the change. You can also enter "nginx -s stop" and "nginx" if the auto restart failed.

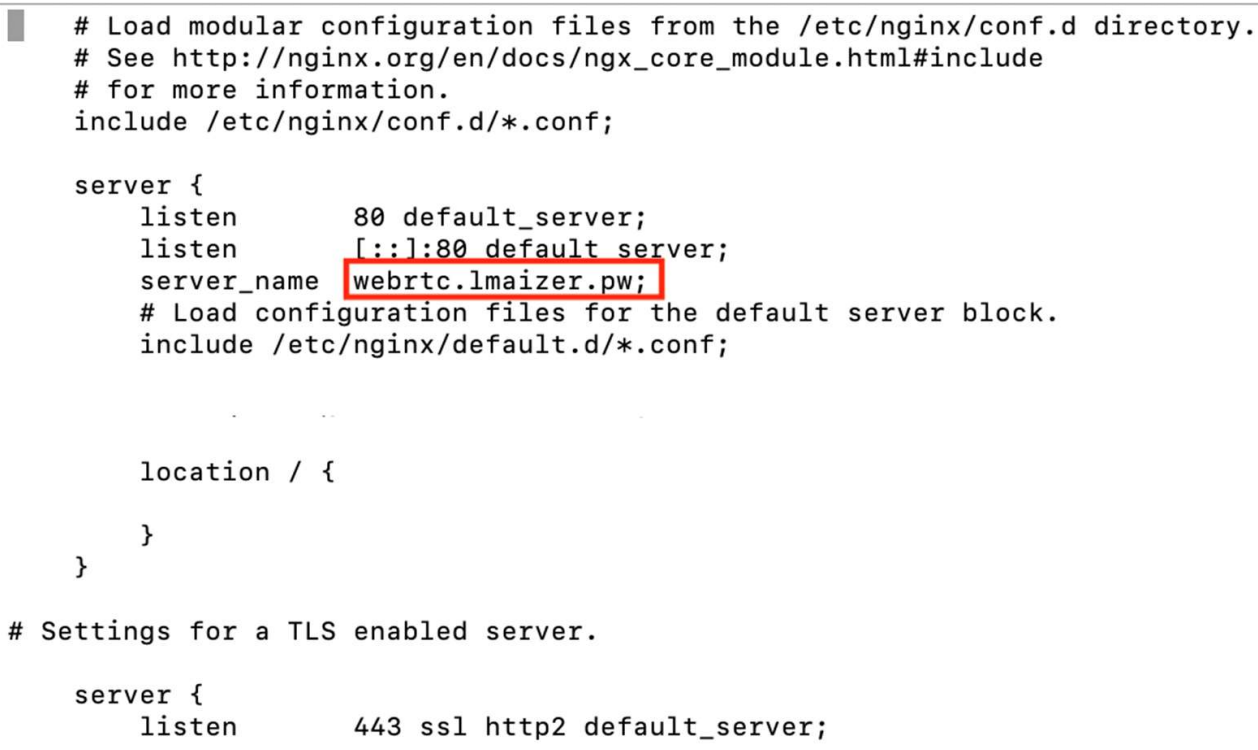


Now, try to visit 'http://:80' this time. If you don't see a 404 not found, you are good. With an http site running on the 80 port of your server, you can apply a free ssl certificate with certbot. Install certbot with "yum -y install yum-utils" and "yum-config-manager --enable rhui-REGION-rhelserver-extras rhui-REGION-rhel-server-optional". The run certbot with "certbot certonly -webroot". Enter all the information needed following certbot's instructions. Certbot would inform you the location of your certificate after finished.

After you get your ssl certificate, include the certificate in nginx. Edit /etc/nginx/nginx.conf again. This time, find the 'server' section, which listens port 443. Put your domain name, as well as the location of your ssl certificate (see red rectangle). You also need to set up a local proxy to let users visit WebRTC page instead of the default nginx page (see blue rectangle).

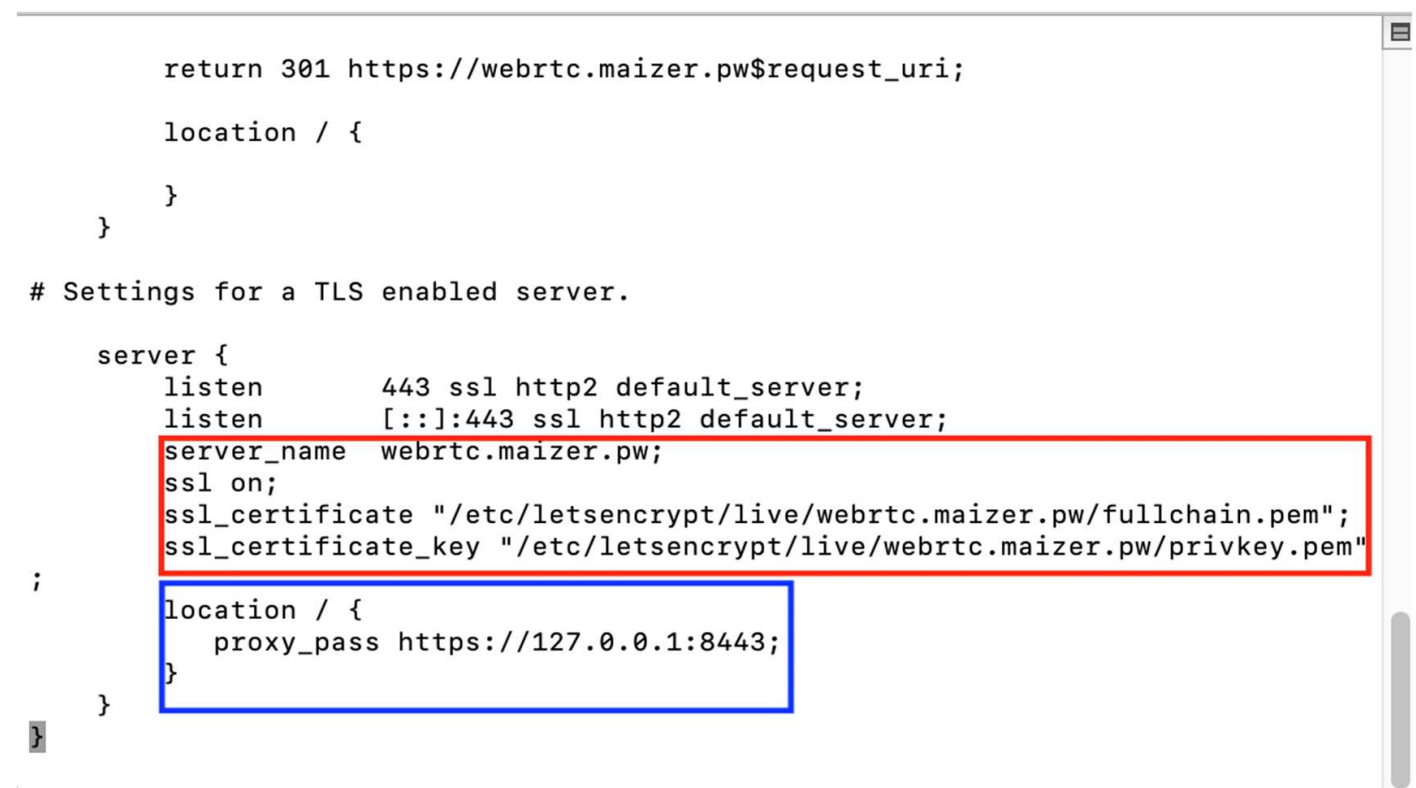

In addition, you want every user to connect to your server using https protocol instead of http. Therefore, go back to the server section, which listens port 80 and set up a 301 redirection. 


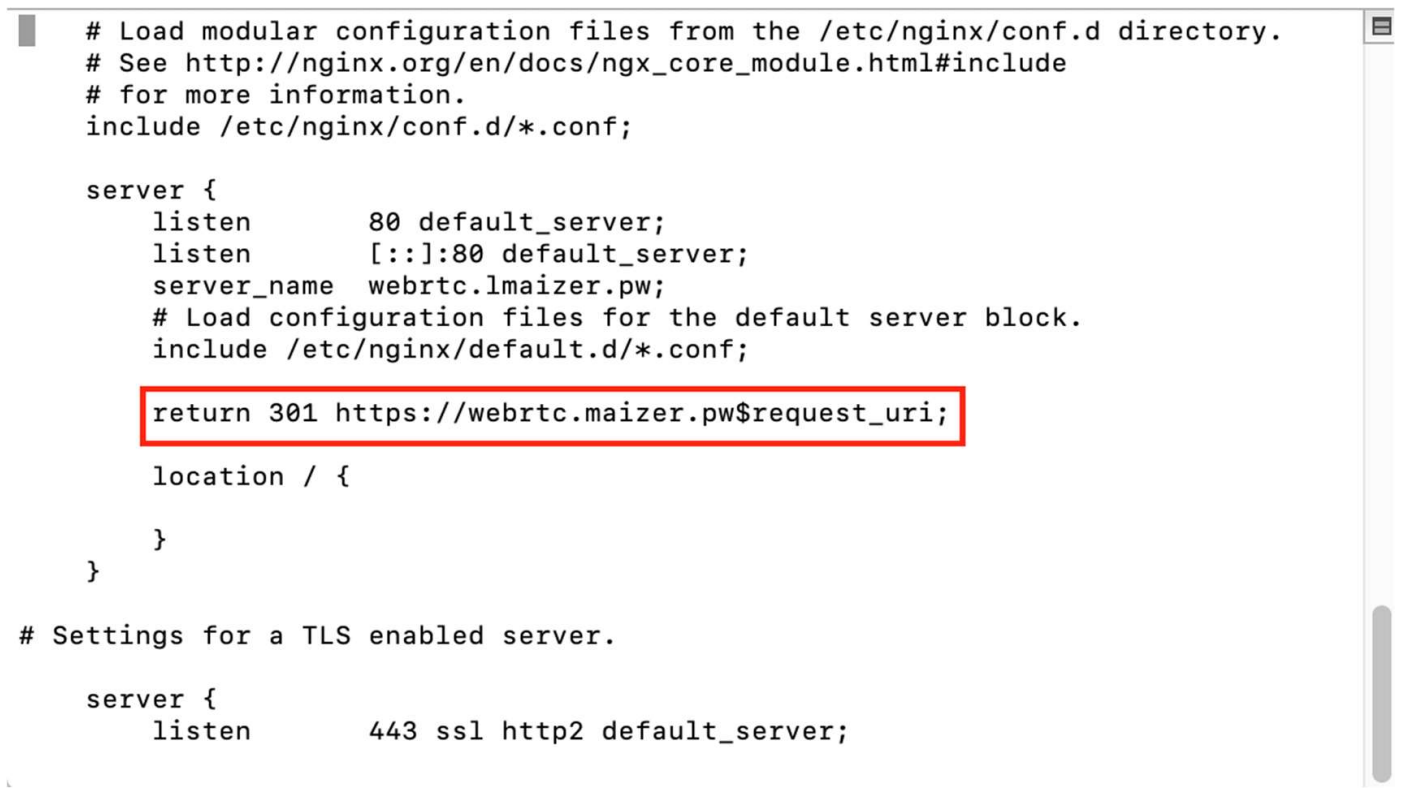

\section{Final step}

After you set up https, your WebRTC server is ready to go. Try visiting the website that you have just built using a device with a microphone and test whether the live streaming is working or not.

\section{Note S3. VR streaming through mainstream platform Twitch.com}

Twitch is one of the mainstream platforms for broadcasting and also supports our 360-degree VR demonstration of chemical experiments. To broadcast on Twitch.com, an extra tool (OBS Studio) needs to be downloaded. After OBS Studio is open, click "Settings" and find "Stream" in the menu. Then click the "Connect Account" button and log in your Twitch account or use stream key to broadcast. 


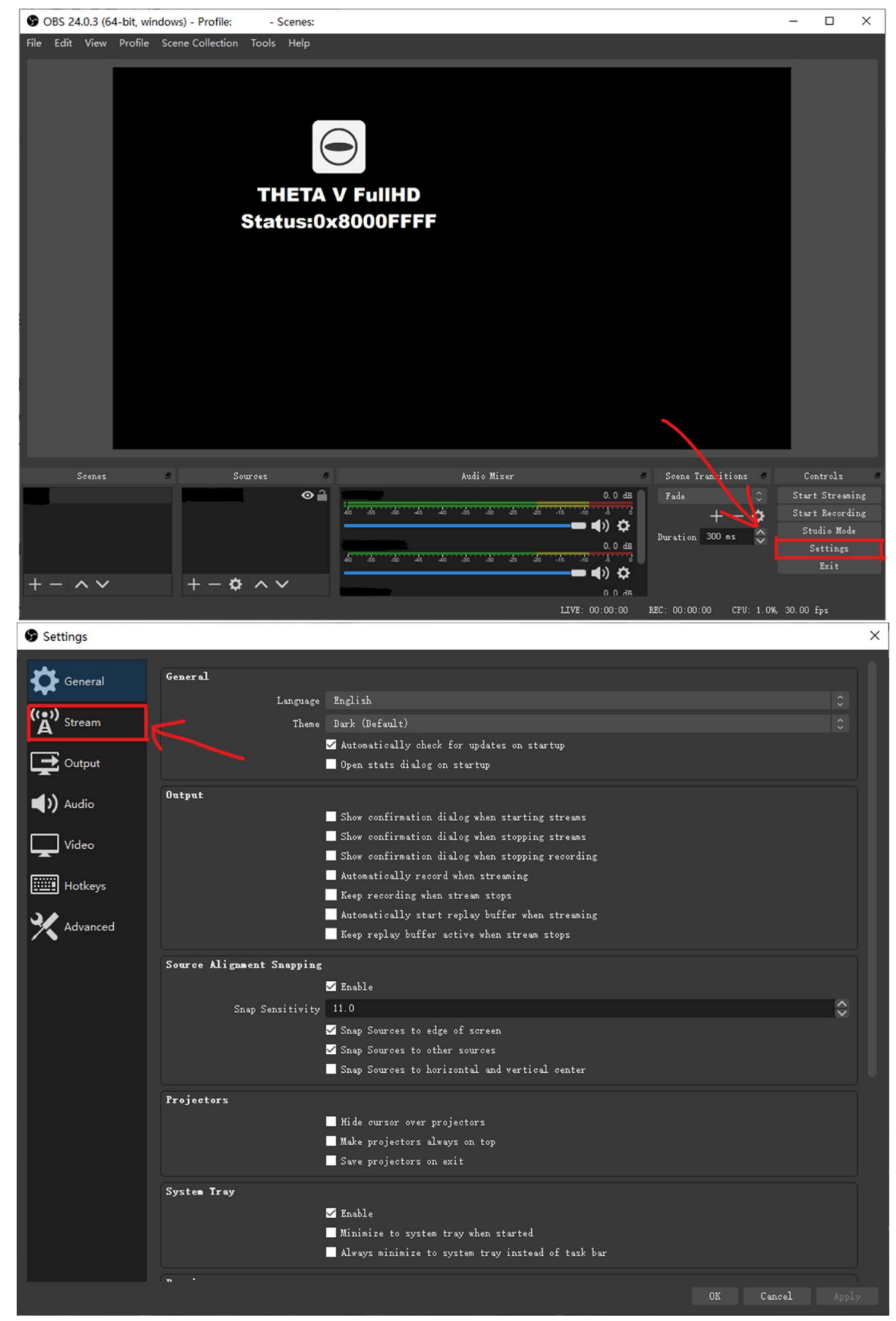




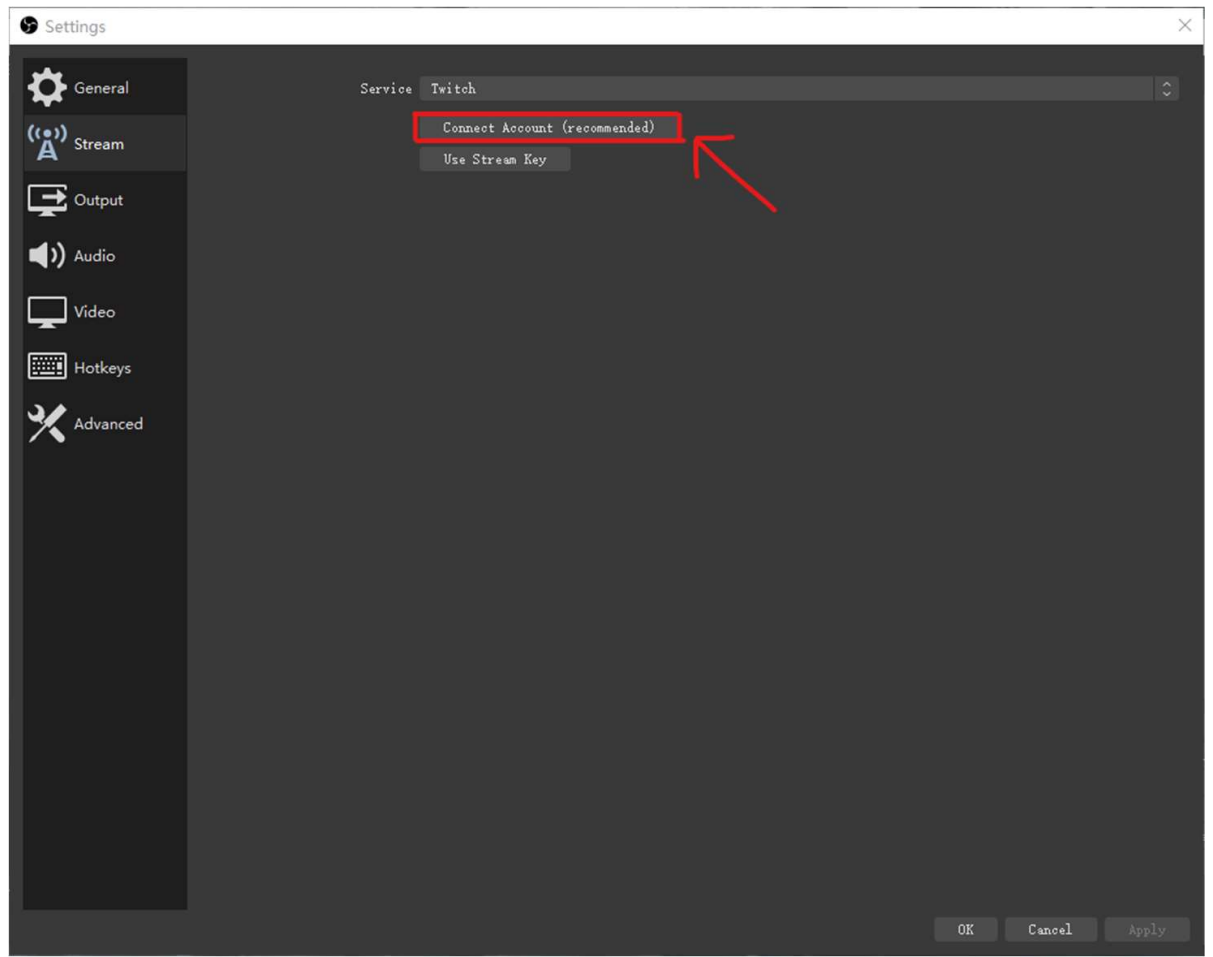

\section{References}

[1] "Real time communication with WebRTC" [Online].

https://codelabs.developers.google.com/codelabs/webrtc-web/\#0. [Accessed: 18-Dec-2019]. [2] "Videojs-panorama", Videojs-panorama by Wensheng Yan [Online]. https://yanwsh.github.io/videojspanorama/. [Accessed: 18-Dec-2019]. 